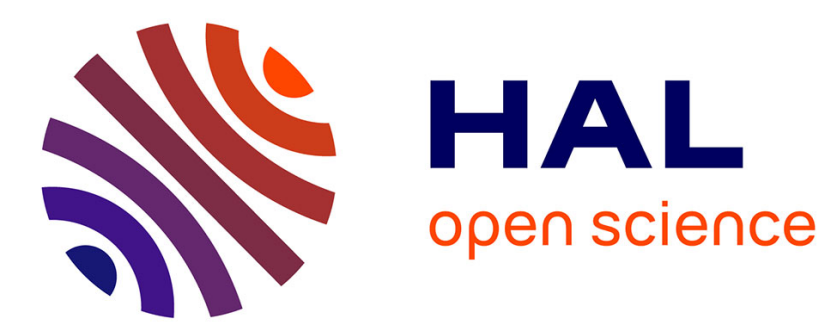

\title{
Cutting force sensor based on digital image correlation for segmented chip formation analysis
}

Thomas Baizeau, Sébastien Campocasso, Frédéric Rossi, Gérard Poulachon, François Hild

\section{- To cite this version:}

Thomas Baizeau, Sébastien Campocasso, Frédéric Rossi, Gérard Poulachon, François Hild. Cutting force sensor based on digital image correlation for segmented chip formation analysis. Journal of Materials Processing Technology, 2016, 238, pp.466-473. 10.1016/j.jmatprotec.2016.07.016 . hal01361336

\section{HAL Id: hal-01361336 https://hal.science/hal-01361336}

Submitted on 7 Sep 2016

HAL is a multi-disciplinary open access archive for the deposit and dissemination of scientific research documents, whether they are published or not. The documents may come from teaching and research institutions in France or abroad, or from public or private research centers.
L'archive ouverte pluridisciplinaire HAL, est destinée au dépôt et à la diffusion de documents scientifiques de niveau recherche, publiés ou non, émanant des établissements d'enseignement et de recherche français ou étrangers, des laboratoires publics ou privés. 


\title{
Cutting force sensor based on digital image correlation for segmented chip formation analysis
}

\author{
Thomas Baizeau ${ }^{\mathrm{a}, *}$, Sébastien Campocasso ${ }^{\mathrm{a}, \mathrm{b}}$, Frédéric Rossi ${ }^{\mathrm{a}}$, Gérard Poulachon ${ }^{\mathrm{a}}$, \\ François Hild ${ }^{\mathrm{c}}$ \\ a Arts et Metiers ParisTech, LaBoMaP, Rue Porte de Paris, 71250 Cluny, France \\ b CEA, DAM, Valduc, 21120 Is-sur-Tille, France \\ ${ }^{c}$ LMT-Cachan, ENS Cachan/CNRS/Université Paris-Saclay 6, 61 avenue du Président Wilson, 94235 Cachan Cedex, France
}

\section{A R T I C L E I N F O}

\section{Article history:}

Received 18 January 2016

Received in revised form 5 July 2016

Accepted 11 July 2016

Available online 25 July 2016

\section{Keywords:}

Chip formation

Cutting forces

Digital image correlation

Analytical modeling

\begin{abstract}
A B S T R A C T
Conventional piezoelectric sensors cannot record the force fluctuations at high frequencies to monitor serrated chip formation. Recently, force measurements by using digital image correlation (DIC) have been reported thanks to imaging devices that become more and more efficient, thereby opening possibilities of high rate acquisition. This study proposes to apply DIC based on closed-form solutions in order to measure cutting forces at camera acquisition frequency. The considered displacement fields are obtained from the Flamant-Boussinesq solution. This method is first applied to picture pairs shot during the cut and then to a full sequence of pictures recorded upon orthogonal cutting of hardened AISI 52100 steel with a c-BN tool. To validate part of the corresponding mechanism, the change of cutting forces is finally investigated when chip segments are formed.
\end{abstract}

\section{Introduction}

In the case of hard machining, serrated chips are produced at high segmentation rate up to $30 \mathrm{kHz}$ (Poulachon and Moisan, 2000). Therefore the change of cutting forces during chip segment formation cannot be recorded by conventional force sensors. Due to their quartz cells, the mechanical bandwidth is commonly of the order of $4 \mathrm{kHz}$ and decreases with the mass on the sensor. This lack of information leads the authors to considerer the mean forces recorded for surface integrity predictions. The numerical model (Umbrello and Rotella, 2012) or the mechanistic cutting forces prediction(Ren and Altintas, 2000) are identified with the mean force levels. Coupled with metallurgical and thermal considerations, Chou and Song (2003) explained the influences of both forces and temperature on the so-called "white layer" formation. However these simulations only considered continuous external loadings (i.e., forces and thermal flux) as in Oxley's predictive machining theory (Oxley, 1989). Yang and Liu (2015) employed a predictive cutting force model in order to determine a plastically deformed area inside the workpiece by solving the Flamant-Boussinesq analytical problem for kinematic fields and Hooke's law for stress calculation. However,

\footnotetext{
* Corresponding author.

E-mail address: thomas.baizeau@ensam.eu (T. Baizeau).

URL: http://labomap.ensam.eu (T. Baizeau).
}

the suitability of the displacement modeling has not been checked in terms of inner displacements in the material.

With the growth of computer performances, many numerical models of orthogonal cutting of hard materials have been proposed. Movahhedy et al. (2002) exposed the existence of a dead zone of material in front of a negative chamfered c-BN tool, which is commonly used in hard machining, with an ALE thermomechanical modeling. Only continuous chips are simulated whereas hard cutting mostly generates serrated chips. Mabrouki and Rigal (2006) investigated thermal softening influences on chip formation of hardened AISI 4340 steel and succeeded in simulating a serrated chip. The cutting force extracted from the simulation presented oscillations with an amplitude of about $16 \%$ of the mean level. Yet no comparison with experimentally measured cutting forces was performed. High frequency cutting force measurements are desirable for simulation or modeling improvements.

From an experimental point of view, digital image correlation (DIC) is appealing in the case of machining thanks to the development of high-speed optical sensors. In terms of frame rate, exposure time, resolution and noise, high speed imaging devices are now efficient for recording images of cutting operations at commonly used cutting speed $V_{c}$ and uncut chip thickness $h$ conditions (e.g., $2.5 \leq V_{c} \leq 5 \mathrm{~m} / \mathrm{s}$ Pujana et al., 2008). However, to get accurate measurements with higher spatial resolutions of the strain fields, the experimental configuration and the full acquisition device employed are critical. Arriola et al. (2011) set-up a fixed tool 
experimentation, as most works, thus avoiding synchronization issues between the imaging device and the cutting test. Unfortunately, this configuration mostly leads to blurred images of the workpiece that only enable for primary shear zone analysis. In all experimental configurations, the DIC analysis is performed with side observations of the specimen during orthogonal cutting focusing on small zones to be consistent with the depth of field limitations.

In solid mechanics, full field measurements and identification procedures are widely used (Sutton et al., 2009; Grédiac and Hild, 2012). Two types of DIC approaches have been developed over the years. The first one, which is referred to as local, consists of independently registering small interrogation windows. The information that is kept is the mean displacement of the window, which is assigned to its center (Sutton et al., 2009). The second approach, which is referred to global, registers the whole region of interest (Hild and Roux, 2012a). One of its advantages lies in the fact that direct couplings can be obtained with finite element simulations, for instance, for identification purposes (Roux and Hild, 2008). If kinematic closed-form solutions are available, the measurement and identification steps can be performed in one single analysis by resorting to so-called integrated approaches (Roux and Hild, 2006).

In a previous communication an imaging device synchronized with a milling machine has been introduced allowing the specimen to be motionless while the tool is moving in a planing operation (Baizeau et al., 2015). The present study includes the two previously discussed needs, namely, confirm the analytical model for displacement estimation, and then perform cutting force measurements in the case of orthogonal cutting of hardened AISI 52100 steel. DIC will be used as a load sensor by resorting to the Flamant-Boussinesq solution. It is an extension of the concept of "stress gauge" (Roux et al., 2005). Two different but complementary cameras are utilized sequentially. The first one, producing picture pairs, aims to confirm the selected analytical model. The second one is used to record a full movie of the cut but with lower accuracy.

After introducing the problem in Section 2, the Flamant-Boussinesq elementary solution for cutting force measurement is presented. This model is first identified and validated with a measured displacement field via global DIC. The model is then employed as kinematic basis for an integrated approach thereby improving the overall performances (Section3). After detailing the experimental set-up used to record pictures that are processed to measure cutting forces, both techniques (i.e., the identification by post-processing measured displacement fields and integrated DIC) are compared and limits are presented. Section 4 reports cutting force measurements during serrated chip formation recorded by the high speed camera and validates some of the theories on serrated chip formation during hard cutting.

\section{Problem description}

The studied case is a planing operation performed on a 56 HRC $_{150}$ hardened AISI 52100 bearing steel (ISO:683-17, 2014) with a Arno AH7510 grade c-BN insert (reference CNGA120408 FN). The cutting edge radius is measured at $r_{n}=23.6 \pm 0.9 \mu \mathrm{m}$ with focus variation measurement. A specific tool holder, presented by Baizeau et al. (2015), that ensures a clearance angle $\alpha_{n}=7^{\circ}$ and a rake angle $\gamma_{n}=7^{\circ}$, is used. It also adjusts the insert orientation so that a rectilinear portion of the cutting edge is employed to cut the specimen in orthogonal cutting condition (planing). The specimen thickness $b=2.5 \mathrm{~mm}$ was chosen to be smaller than the c-BN insert. In this particular configuration (see Fig. 1), the cutting force $F_{c}(\mathrm{~N})$ in the $x$-direction can be considered as the resultant normal force studied by Flamant (1892). The author expressed the

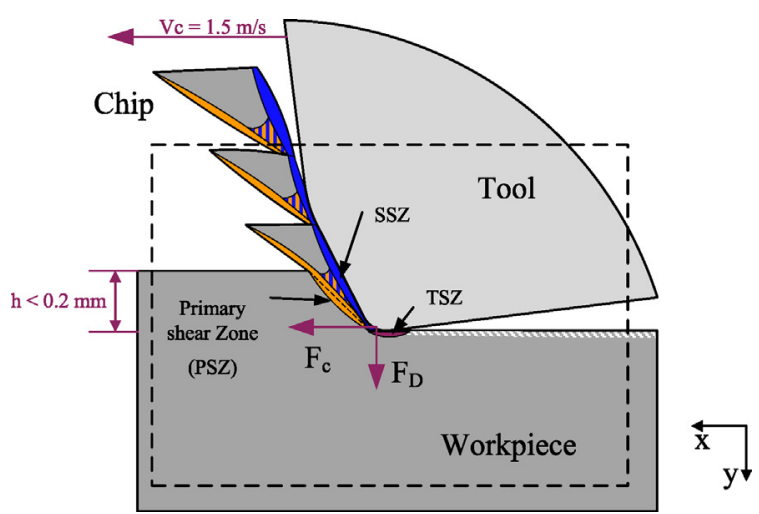

Fig. 1. Schematic view of the observed hard planing operation.

displacement field induced by a normal linear load applied to an elastic semi-infinite medium. Boussinesq (1892) generalized the previous results to any loading direction. In a planing configuration, the resultant thrust force $F_{D}(\mathrm{~N})$ is acting as the orthogonal load (i.e., along the $y$-direction). Therefore, an identification of the load components is proposed starting from the measured displacement fields and assuming that the material is elastic.

\subsection{Flamant-Boussinesq solution}

The Flamant-Boussinesq solution is first given for plain strain configurations. It is convenient to introduce the complex representation of the considered 2D-space, namely $z=x+i y=r \mathrm{e}^{i \theta}$. The coordinate system origin is chosen to be the force application point as shown in Fig. 2.

The stress function (Airy potential) of an orthogonal line force $f_{y}$ $(\mathrm{N} / \mathrm{mm})$ acting along a straight boundary with a width $b(\mathrm{~mm})$ was expressed by Flamant (1892)

$\phi_{f_{y}}(\boldsymbol{z})=\frac{f_{y}}{\pi} r \theta \cos (\theta)$

The stress distribution is then obtained by integrating in the space $(\theta \in[-(\pi / 2),(\pi / 2)])$. Considering an elastic behavior of the material, the strains and displacements are computed. In order to determine integration constants, it is assumed that:

- any point $M(r, \theta=0)$ in the loading direction $(\overrightarrow{e y})$ has no tangential motion: $u(M(r, \theta=0))=0 \cdot \overrightarrow{e x}+u_{y} \cdot \overrightarrow{e y}$,

- the point $M(r=d, \theta=0)$ has no radial motion: $u(M(r=d, \theta=0))=$ $0 \cdot \overrightarrow{e x}+0 \cdot \overrightarrow{e y}$

- $d$ is large in terms of St Venant principle with respect to the studied area.

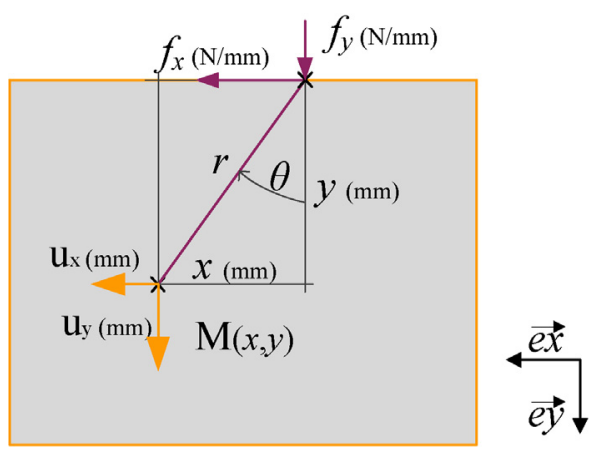

Fig. 2. Parameterization of Flamant-Boussinesq problem. 
The same calculation has been performed by Boussinesq (1892) with a tangential line force $f_{x}(\mathrm{~N} / \mathrm{mm})$ for which the stress function becomes

$\phi_{f_{x}}(\boldsymbol{z})=\frac{f_{x}}{\pi} r\left(\frac{\pi}{2}+\theta\right) \cos \left(\frac{\pi}{2}+\theta\right)$

Last, the total displacement field $\boldsymbol{u}_{F B}$ induced by any force combination in the material is expressed by superposition of the displacements induced by the normal and tangential forces

$\boldsymbol{u}_{F B}=\boldsymbol{u}_{f_{x}}+\boldsymbol{u}_{f_{y}}$

\subsection{Integrated approach}

Another approach is to identify directly the parameter vector $\{\boldsymbol{p}\}$ by resorting to integrated DIC (Roux and Hild, 2006). Contrary to the previously presented identification procedure, the kinematic basis for displacement measurements consists of the Flamant-Boussinesq fields in addition to the rigid body motions. The gray level conservation principle is applied between a reference image $I$ and a deformed image $J$. The sum of squared gray level differences

with

$\boldsymbol{u}_{f_{x}}=\frac{f_{x}}{2 \pi \mu}\left[\left(\frac{y^{2}}{x^{2}+y^{2}}+\frac{\lambda+2 \mu}{\lambda+\mu} \log \frac{b}{\sqrt{x^{2}+y^{2}}}\right)+i\left(\frac{x y}{x^{2}+y^{2}}-\frac{\mu}{\lambda+\mu} \arctan \frac{x}{y}\right)\right]$

$\boldsymbol{u}_{f_{y}}=\frac{f_{y}}{2 \pi \mu}\left[\left(\frac{x y}{x^{2}+y^{2}}+\frac{\mu}{\lambda+\mu} \arctan \frac{x}{y}\right)+i\left(\frac{x^{2}}{x^{2}+y^{2}}-\frac{\mu}{\lambda+\mu}+\frac{\lambda+2 \mu}{\lambda+\mu} \log \frac{b}{\sqrt{x^{2}+y^{2}}}\right)\right]$

where $\lambda$ and $\mu$ are Lamé's coefficients.

\subsection{Model identification with measured kinematic field}

Let $\left\{\boldsymbol{u}_{m}\right\}^{t}=\left\{\boldsymbol{u}_{1}, \ldots, \boldsymbol{u}_{n}, \ldots, \boldsymbol{u}_{N}\right\}$ be measured displacements on the specimen at a number of points $M_{n}\left(z_{n}\right)$ with $n=1, \ldots, N$. In the present work, this field is obtained by Q4-DIC over a regular mesh with a global approach using 4-noded elements (Besnard et al., 2006). If there is no rigid body motion, the measured displacement should be as close as possible to the Flamant-Boussinesq solution.

$\boldsymbol{u}_{F B}\left(\boldsymbol{z}_{n}\right)=f_{x} \boldsymbol{u}_{f_{x}}\left(\boldsymbol{z}_{n}\right)+f_{y} \boldsymbol{u}_{f_{y}}\left(\boldsymbol{z}_{n}\right)$

Because of vibrations for example, rigid body motions occur during the experiment. Therefore rigid body translations $\left(t_{x}, t_{y}\right)$ and rotation $\omega$ have to be added to the kinematic basis

$$
\begin{aligned}
& \boldsymbol{u}_{F B}\left(\boldsymbol{z}_{\boldsymbol{n}}, f_{x}, f_{y}, t_{x}, t_{y}, \omega\right) \\
& \quad=f_{x} \boldsymbol{\psi}_{1}\left(\boldsymbol{z}_{\boldsymbol{n}}\right)+f_{y} \boldsymbol{\psi}_{2}\left(\boldsymbol{z}_{\boldsymbol{n}}\right)+t_{x} \boldsymbol{\psi}_{3}\left(\boldsymbol{z}_{\boldsymbol{n}}\right)+t_{x} \boldsymbol{\psi}_{4}\left(\boldsymbol{z}_{\boldsymbol{n}}\right)+\omega \boldsymbol{\psi}_{5}\left(\boldsymbol{z}_{\boldsymbol{n}}\right)
\end{aligned}
$$

with

$$
\begin{aligned}
& { }_{1}\left(\boldsymbol{z}_{\boldsymbol{n}}\right)=\boldsymbol{u}_{f_{x}}\left(\boldsymbol{z}_{\boldsymbol{n}}\right), \quad \boldsymbol{\psi}_{2}\left(\boldsymbol{z}_{\boldsymbol{n}}\right)=\boldsymbol{u}_{f_{y}}\left(\boldsymbol{z}_{\boldsymbol{n}}\right), \quad \boldsymbol{\psi}_{3}\left(\boldsymbol{z}_{\boldsymbol{n}}\right)=1, \\
& { }_{4}\left(\boldsymbol{z}_{\boldsymbol{n}}\right)=i, \quad \boldsymbol{\psi}_{5}\left(\boldsymbol{z}_{\boldsymbol{n}}\right)=i \boldsymbol{z}_{\boldsymbol{n}}
\end{aligned}
$$

The identification procedure is based upon a least squares procedure. It consists of minimizing the sum of squared differences

$\mathcal{T}\left(f_{x}, f_{y}, t_{x}, t_{y}, \omega\right)=\sum_{n}\left|\boldsymbol{u}_{F B}\left(\boldsymbol{z}_{\boldsymbol{n}}, f_{x}, f_{y}, t_{x}, t_{y}, \omega\right)-\boldsymbol{u}_{n}\right|^{2}$

with respect to the five unknown parameters (i.e., $f_{x}, f_{y}, t_{x}, t_{y}, \omega$ ) gathered in the column vector $\{\boldsymbol{p}\}$, which leads to a linear system of equations

$[\boldsymbol{L}]\{\boldsymbol{p}\}=\{\boldsymbol{b}\}$

with

$L_{i j}=\sum_{n} \mathfrak{R}\left(\boldsymbol{\psi}_{i}\left(\boldsymbol{z}_{\boldsymbol{n}}\right) \boldsymbol{\psi}_{j}\left(\overline{\boldsymbol{z}}_{\boldsymbol{n}}\right)\right), \quad b_{i}=\sum_{n} \mathfrak{R}\left(\boldsymbol{\psi}_{i}\left(\boldsymbol{z}_{\boldsymbol{n}}\right) \overline{\boldsymbol{u}}_{n}\right)$

Last, let us mention that the application point of the load for Flamant-Boussinesq solution (Eq.(5)) is selected as the intersection of the rake face and the flank face of the tool. $\eta\left(f_{x}, f_{y}, t_{x}, t_{y}, \omega\right)=\sum_{R O I}\left[I(\boldsymbol{z})-J\left(\boldsymbol{z}+\boldsymbol{u}_{\mathrm{FB}}\left(\boldsymbol{z}, f_{x}, f_{y}, t_{x}, t_{y}, \omega\right)\right)\right]^{2}$

is minimized over the considered region of interest (ROI) with respect to the five unknown parameters. A Newton-Raphson scheme is followed, which leads to successive linearizations and corrections (Hild and Roux, 2012)

$[\boldsymbol{M}]\{\delta \boldsymbol{p}\}=\{\boldsymbol{b}\}$

with

$M_{i j}=\sum_{R O I} \mathfrak{R}\left(\boldsymbol{\psi}_{i}(\boldsymbol{z}) \nabla \bar{I}(\boldsymbol{z})\right) \mathfrak{R}\left({ }_{j}(\boldsymbol{z}) \nabla \bar{I}(\boldsymbol{z})\right)$
$b_{i}=\sum_{R O I}(I(\boldsymbol{z})-\tilde{J}(\boldsymbol{z})) \mathfrak{R}\left(\boldsymbol{\psi}_{i}(\boldsymbol{z}) \nabla \bar{I}(\boldsymbol{z})\right)$

and $\tilde{J}(\boldsymbol{z})=J\left(\boldsymbol{z}+\boldsymbol{u}_{F B}(\boldsymbol{z},\{\tilde{\boldsymbol{p}}\})\right), \nabla I(\boldsymbol{z})=I_{, X}(\boldsymbol{z})+i I_{y}(\boldsymbol{z})$, where $\{\tilde{\boldsymbol{p}}\}$ is the current estimate of the sought parameters, and $\{\delta \boldsymbol{p}\}$ the parameter corrections. At convergence, one way of checking the quality of the registration is to analyze the gray level residuals

$\rho(\boldsymbol{z})=\left|I(\boldsymbol{z})-J\left(\boldsymbol{z}+\boldsymbol{u}_{F B}(\boldsymbol{z},\{\boldsymbol{p}\})\right)\right|$

and to compare them with acquisition noise. This is a way of detecting model errors (e.g., areas where the hypothesis of elasticity is not valid). By having measured the parameter vector $\{\boldsymbol{p}\}$, the acting forces are known because they are the first and the second component of $\{\boldsymbol{p}\}$. The application point of the load is kept the same for the identification procedure using Q4-DIC measurements and integrated DIC (referred to as I-DIC in the sequel).

\section{Validation of cutting force measurement}

Both approaches of force measurements via the Flamant-Boussinesq solutions have been implemented in MATLAB ${ }^{\circledR}$. The following section presents the experimental set-up used to validate the measurements in the case of planing.

\subsection{Experimental set-up with double-frame sCMOS imager}

The planing operation has been performed along the $x$-axis of a DMG DMC85V 3-axis milling machine having linear motors that enable for a translation speed up to $2 \mathrm{~m} / \mathrm{s}$. A gradual acceleration has been performed in order to minimize vibrations and to reach the targeted cutting speed few centimeters before material contact. The side of the specimen has been observed by a LaVision 


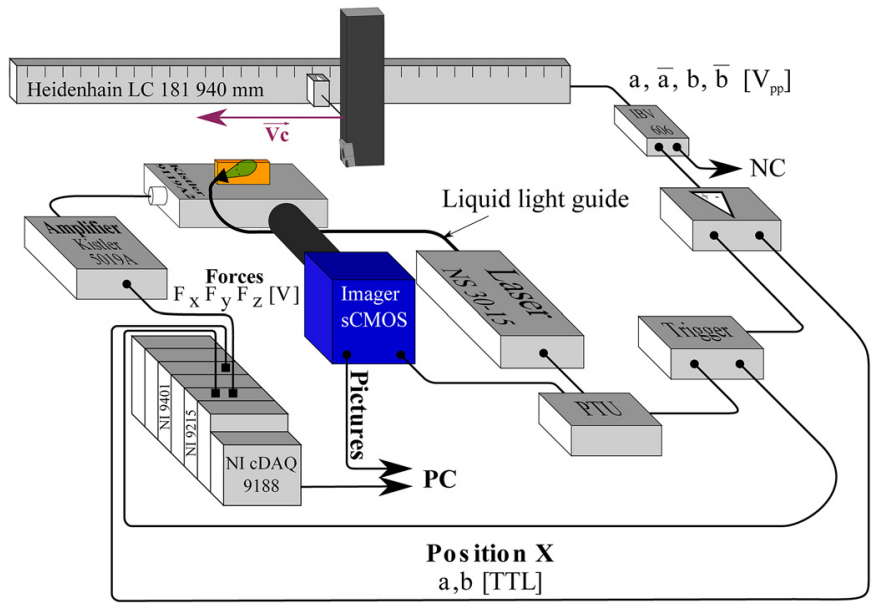

Fig. 3. Principle of the experimental set-up with double frame imager.

sCMOS double-frame imager with a x10 Mitutoyo telecentric lens providing a resolution of $0.66 \mu \mathrm{m} /$ pixel on the $2560 \times 2160$ pixel sCMOS sensor encoded over 16 bits (Fig. 3). Picture pairs composed of two frames ( $f_{0}$ and $f_{1}$ ) are recorded at frequency of $15 \mathrm{~Hz}$ with an adjustable inter-framing time $d t_{i f}$ ranging from $120 \mathrm{~ns}$ to $30 \mathrm{~ms}$ and a minimum exposure time of $10 \mathrm{~ms}$. To get non-blurred images, the scene has been lit by a compact dual-cavity pulsed Nd:YAG $30 \mathrm{~mJ}$ laser (Litron NS 30-15). Each laser performs a $5 \mathrm{~ns}$ flash, which is giving the effective exposure time, delayed by $d t_{i f}$. A liquid light guide ended by an expander is utilized to homogeneously light the scene. The laser head and the imager are synchronized by the Programmable Timing Unit (PTU).

Based on the incremental signal counting of the $x$-linear encoder, a triggering system has been developed to capture the cutting tool in the $1.7 \times 1.4 \mathrm{~mm}$ observed field. A total of three pairs (i.e., 6 frames) have been recorded for each cutting test as presented in Fig. 4. During the experiment, cutting forces $F_{C}(\mathrm{~N})$ and $F_{D}(\mathrm{~N})$ have been monitored by a Kistler 9119AA2 piezoelectric dynamometer and its charge amplifier (Kistler 5019A). Thanks to a NI cDAQ-9188 acquisition device, NI 9215 analog and NI 9401 digital counting acquisition cards, once the cutting forces, the triggering signal of the PTU and the $x$-position have been recorded synchronously with the same time clock allowing $F_{c}(\mathrm{~N}), F_{D}(\mathrm{~N}), x(\mathrm{~mm})$ and $V_{c}(\mathrm{~m} / \mathrm{min})$ to be known at the time of acquisition of the picture pair P2 during the cutting process. Two other picture pairs are also recorded, namely, one before the cut (P1) and one at the end (P3).

After having flattened the upper side of the specimen with unrecorded planing operations, the orthogonal cutting test has been performed ten times in the same configuration with $V_{c}=1.5 \mathrm{~m} / \mathrm{s}, h=0.1 \mathrm{~mm}, b=2.5 \mathrm{~mm}$ over a length of $40 \mathrm{~mm}$ (i.e., length of the specimen).

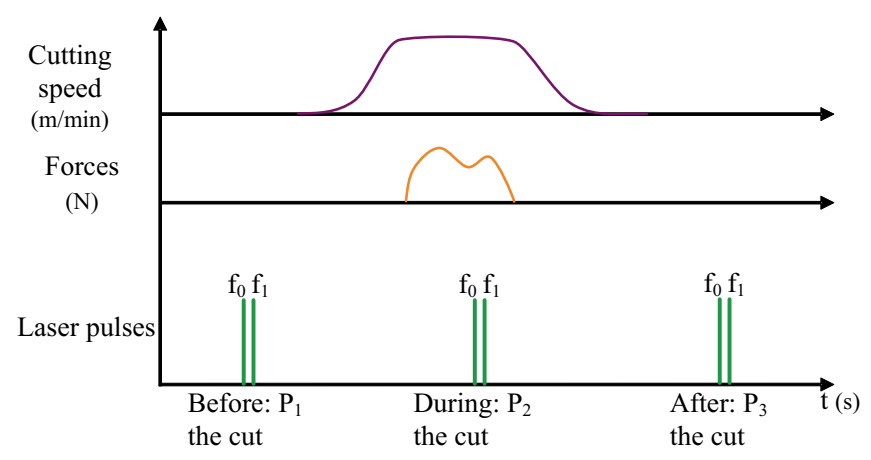

Fig. 4. Cutting test chronogram.

\subsection{Force and kinematic measurement during hard cutting}

For the force measurement or the kinematic field analysis, DIC is performed between a reference image $I$ and a deformed image $J$ that are respectively $P 1_{f_{0}}$ and $P 2_{f_{0}}$. The application point of the load is selected as the intersection of the rake face and the flank face of the tool on picture $J$. For the Flamant-Boussinesq fields, the elastic properties of the material are set to $E=210 \mathrm{GPa}$ for the Young's modulus and $v=0.3$ for the Poisson's ratio (Eq. (4)). The DIC analyses are then performed for a ROI selected in the material which behaves elastically (i.e., few dozen micrometers under the cutting edge).

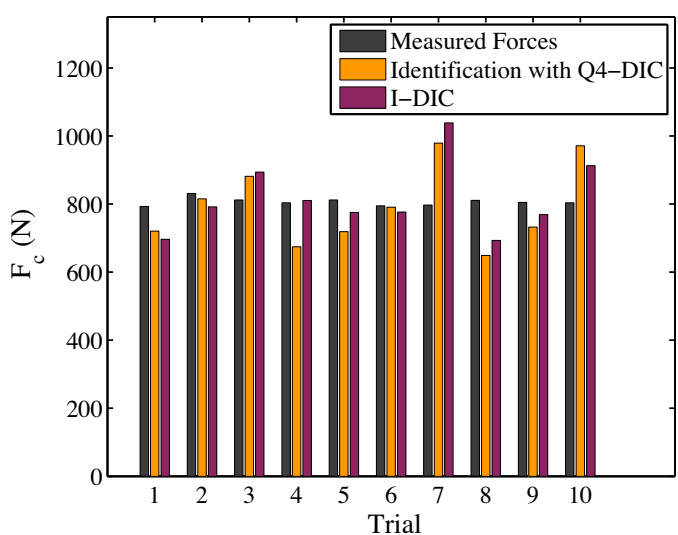

(a) Cutting force

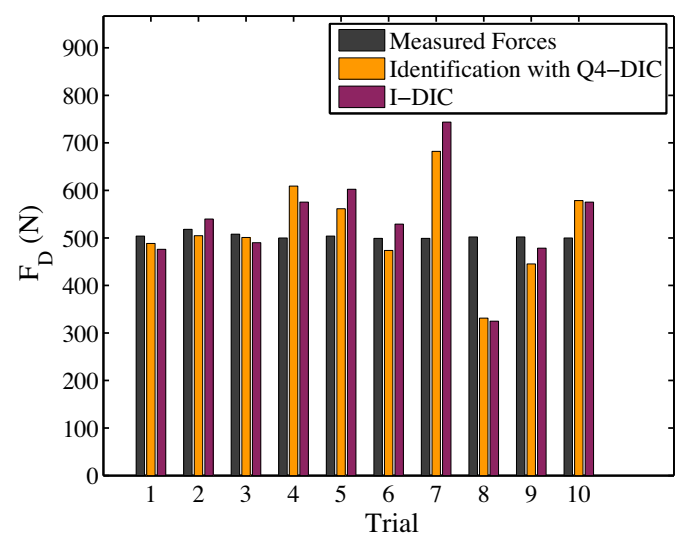

(b) Thrust force

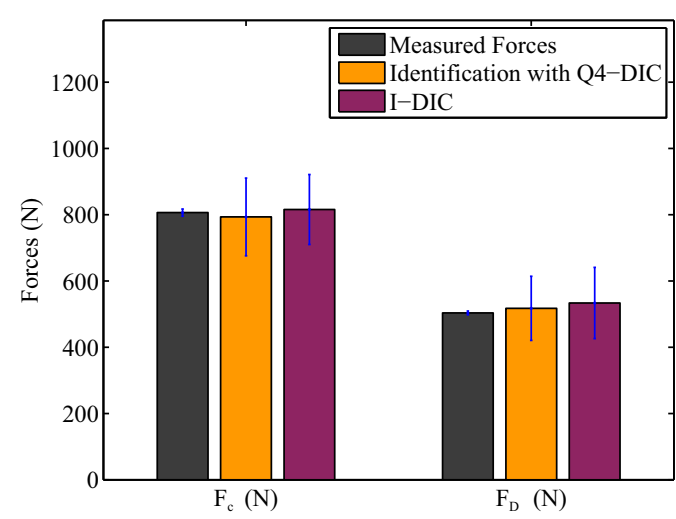

(c) Mean values

Fig. 5. Cutting force measurements: (a) cutting force, (b) thrust force, (c) mean values over ten trials. 


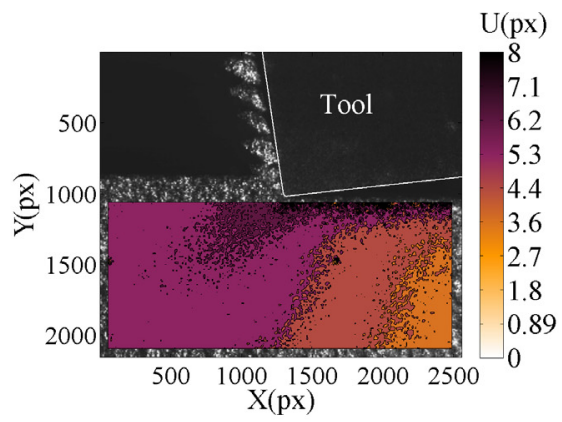

(a) Displacement measured by Q4-DIC

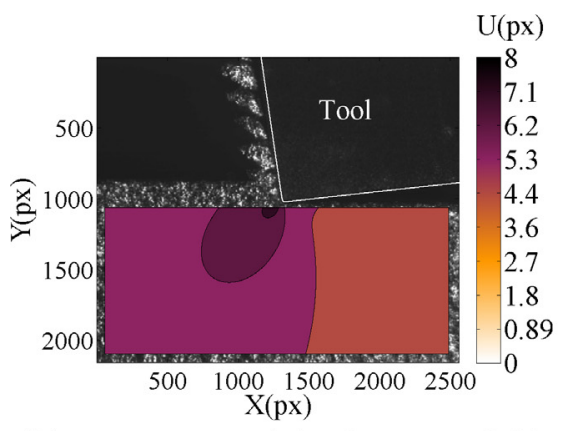

(b) Post-processed displacement field

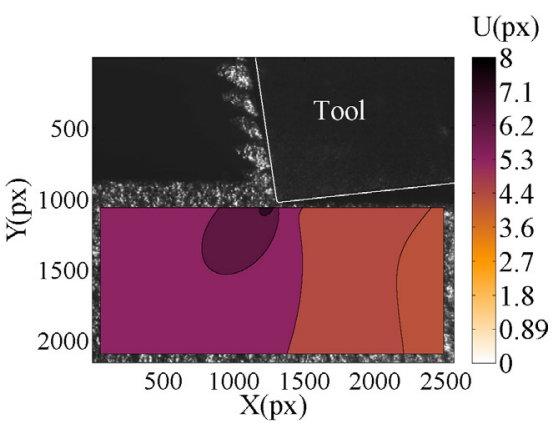

(c) Displacement measured with I-DIC

Fig. 6. Analysis of trial 6. Displacement field (a) measured with Q4-DIC, (b) estimated by subsequent identification, (c) measured by integrated DIC.

The estimated levels of the cutting forces with both approaches using the Flamant-Boussinesq basis are in good agreement for each trial (see Fig. 5a and b). This result cross-validates both identification routes based on pictures only. However, deviations appear from the mean value of the conventionally measured cutting forces. For example an overestimation up to $40 \%$ is observed for trial 7 in the case of the thrust force and up to $30 \%$ for the same trial in the case of the force in the cutting direction. These differences can be explained with observation proposed in Section 4: although the timing of the picture is well mastered, the instant of the segment formation during the picture is not controlled. That leads to high fluctuations on the force measurement by I-DIC that the dynamometer is not able to record.

By analyzing the mean cutting forces (Fig. 5c), the levels estimated by the developed methods are in very good agreement with the dynamometer readings. A higher deviation about the mean value for both the cutting force and the thrust force is still obtained. Less than $1 \%$ and $5 \%$ error for respectively the cutting force and the penetration force are obtained with I-DIC. These results fully validate the integrated approach.

In terms of kinematic field, the relevance of the Flamant-Boussinesq model is validated by comparing the Q4-DIC measurements (Fig. 6a) for which no mechanical statement is made apart from the continuity requirement of the displacements and the identification results shown in Fig. 6b. The latter ones are themselves very close to the measurements provided by I-DIC (Fig. 6c). This constitutes an additional full-field validation of the proposed framework.

The map presented in Fig. 7a shows the relative error

Relativeerror $=\frac{\left|\boldsymbol{u}_{F B}-\boldsymbol{u}_{Q 4}\right|}{\boldsymbol{u}_{\mathrm{Q} 4}}(\%)$

of the displacement estimation obtained by the Flamant-Boussinesq model with respect to Q4-DIC measurements. A mean error of $5.1 \%$ is found for the studied case. The displacement field measured by the integrated approach (Fig. 6c) is closer to Q4-DIC results since the error map shown in Fig. 7b leads to a mean value of about $4 \%$. This is due to the fact that the least squares technique used herein to post-process the measured fields is not weighted by the covariance matrix associated with the uncertainty of the measured degrees of freedom. Conversely, integrated DIC automatically weights the measurements since all pixels are considered on an equal footing (Mathieu et al., 2015).

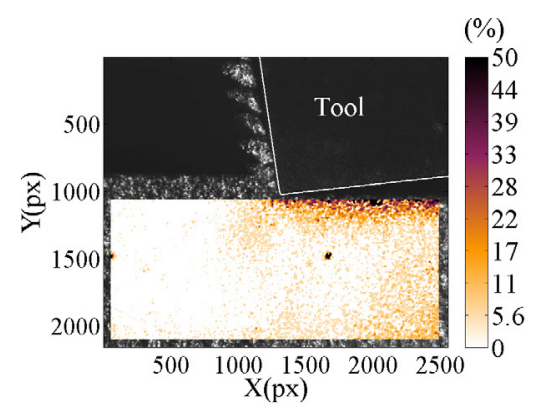

(a)

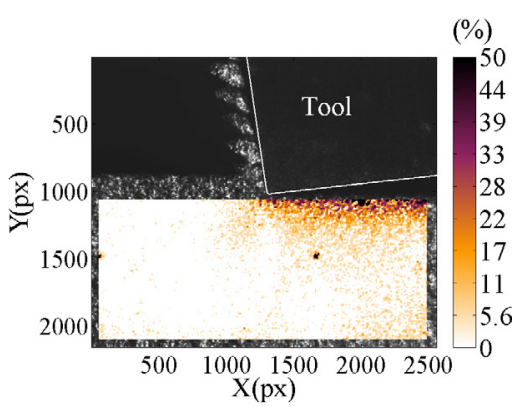

(b)

Fig. 7. Analysis of trial 6. (a) Relative error map of the identification procedure vs. Q4-DIC. (b) Relative error map of I-DIC vs. Q4-DIC. 
In both methods, the displacement error increases at the back of the cutting edge. This can be explained by the difficulty of performing Q4-DIC (and I-DIC) since the gray level conservation is not fully satisfied close to the machined surface (Baizeau et al., 2015). The integrated approach proposed herein will be preferred in order to avoid the identification step of Q4-DIC measurements. With all the previous discussions, the ability of cutting force measurement via integrated DIC based on the Flamant-Boussinesq solution is validated. However there are also some limitations that are exposed in the following section.

\subsection{Measurement limitations}

Despite the fact that the developed method is completely integrated, it cannot be performed if the cutting forces are so small or the Young's modulus so high that they generate displacements of the same order of magnitude as the measurement uncertainties due to the whole acquisition device (e.g., camera electrical noise, lighting, machine-tool vibrations). This standard uncertainty level is of the order of one tenth of a pixel for Q4 elements of edge size $\ell=8$ pixels. It is calculated by Q4-DIC with the two pictures $P 1_{f_{0}}$ and $P 1_{f_{1}}$ prior to loading and therefore corresponds to the standard resolution. This resolution level explains the high frequency fluctuations that can be seen in Fig. 6 a.

A good knowledge of the elastic material properties is also a key information for an accurate estimation of the cutting forces. As shown in Eq. (4), the displacements and the forces are proportional to the Young's modulus. Having misestimated the Young's modulus directly impacts the results. The fact that the mean force levels measured by the dynamometer and both DIC approaches are very close validates the value of the Young's modulus.

Finally, a dual frame imager with a low repetition rate (i.e., the presented case) can be employed for recording cutting tests with serrated chip but the lack of control of the instant of chip formation led us to use a high speed camera. With the use of the integrated approach, which is faster than the identification with Q4-DIC fields, this type of chip formation is studied with I-DIC.

\section{Serrated chip formation analysis}

In the case of hard machining, serrated chips are produced at high frequency. To analyze the chip formation, multiple images are needed during a chip segment formation. For this purpose, a second experiment has been developed.

\subsection{Experimental set-up with high speed camera}

The experimental environment used is the same as presented in Section 3.1. Only the lighting source, the camera and the PTU have changed. An ultra-high speed camera Photron Fastcam SA-Z with a 12-bit monochrome CMOS sensor has been mounted in the milling machine. A high power LED lighting source has been specially developed to get clear pictures even at $1 \mathrm{Mfps}$. The triggering device is kept the same and is directly working with the camera without any PTU interface. The same experimental sequence as previously mentioned (Section 3.1) is followed. However, to improve the quality of the analyze, a lower cutting speed: $V_{c}=1 \mathrm{~m} / \mathrm{s}$, is chosen. Therefore more picture are captured during a segment formation.

The number of pictures recorded during a cutting test is set in order to get multiple images during a chip segment formation. The selected frame rate is $50,000 \mathrm{fps}$ with an exposure time of $10 \mu \mathrm{s}$. Two sequences of 100 images have been recorded. The first sequence before the process (i.e., unloaded configuration), and the second during the planing test (corresponding to the whole pass of
Forces "measured" by I-DIC

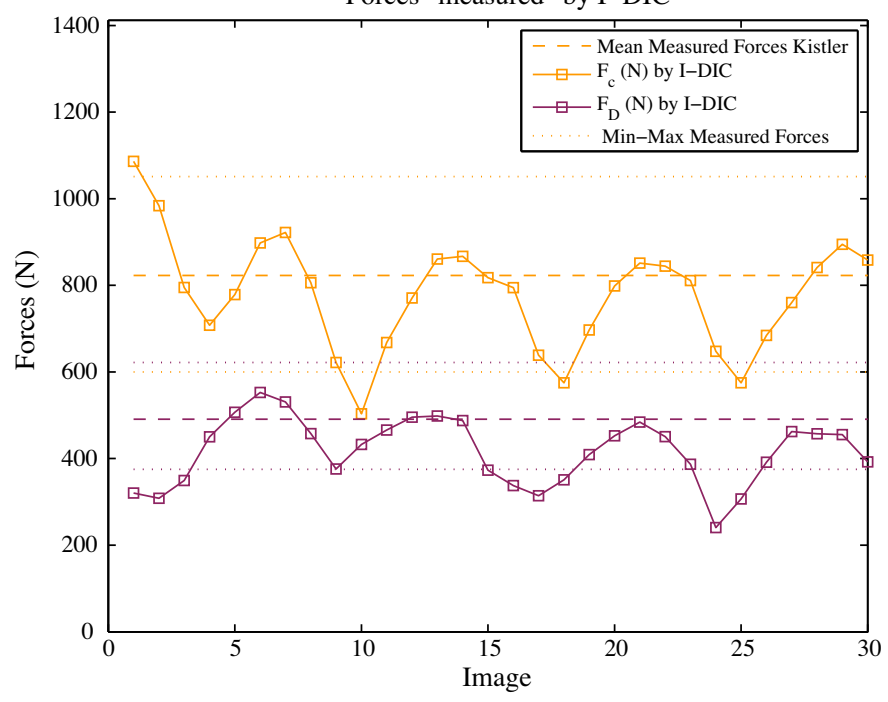

Fig. 8. Evolution of cutting forces during 4 segments formation; $V_{c}=1 \mathrm{~m} / \mathrm{s}$, $h=0.10 \mathrm{~mm}$.

the cutting edge in the scene). In the second sequence, 30 successive pictures $\left\{J_{1}, \ldots, J_{i}, \ldots, J_{30}\right\}$ have been selected to be analyzed with the developed I-DIC approach. Each image is registered with a reference image $I$ chosen in the first sequence of 100 pictures. The $J_{i}$ images correspond to the generation of 4 segments with the tool crossing through the middle of the pictures.

The determination of the force application point has been performed the same way as previously mentioned (Section 3.2) on the first picture $\left(J_{1}\right)$ of the set of deformed images. Due to the displacement of the tool, this application point has to be recalculated for each image. An automatic procedure has been developed. The rigid body motion of the tool is measured with standard Q4-DIC between the deformed images $J_{i}$ with $i \in[2, \ldots, 30]$ and the first deformed image $J_{1}$. Each value of the tool motion is then applied to the previously determined force application location in order to move this point for every deformed image. Last, I-DIC calculations between each picture $J_{i}$ and the reference picture $I$ are performed with the determined force application point in order to measure the instantaneous cutting force and the corresponding displacement field.

\subsection{Cutting force during chip segments formation}

The change of the cutting forces measured by I-DIC approach is reported in Fig. 8. A periodic evolution of the forces is visible with a frequency of $6.9 \mathrm{kHz}$ and an amplitude of $130 \mathrm{~N}$ and $90 \mathrm{~N}$ for respectively the cutting and thrust forces. This amplitude represents about $18 \%$ of the mean value during the test for the cutting force and $22 \%$ for the thrust force.

Each time the cutting force reaches a minimum value, a chip segment is completely formed as observed in images 9 (Fig. 9a, segment number $n-1$ ) and 17 (Fig. 9i, segment number $n$ ). While the segment $n-1$ is moving out (see image 10, Fig. 9b), the cutter is moving forward and pushes on the remaining plastic part of the $45^{\circ}$ shear plane previously formed (for $n-1$ segment), thereby increasing slowly the cutting force. In images 11,12 and 13 (see Fig. $9 \mathrm{c}-\mathrm{e}$ ), the undamaged material behind the $n-1$ shear plane is compressed by the cutter without any out-of-plane distortions in the future segment. The cutting forces reach a maximum level at image 14 (Fig. 9f), and simultaneously the material bulges over a triangle that will become the segment $n$. The loading applied by the cutting tool induces yielding of the material that starts shearing along a plane oriented at an angle of $45^{\circ}$ between the tool tip 


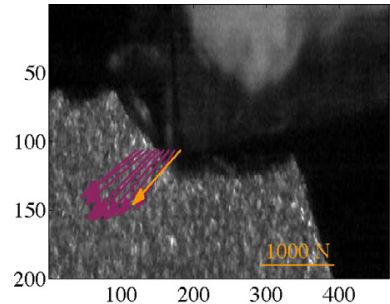

(a) image 9

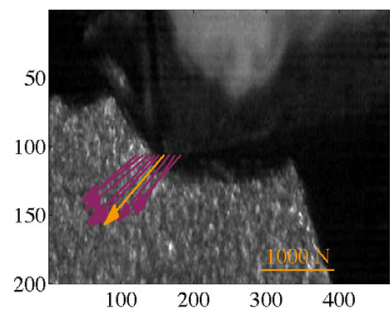

(d) image 12

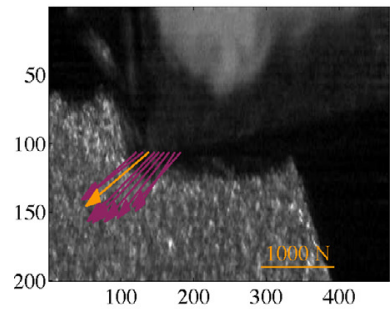

(g) image 15

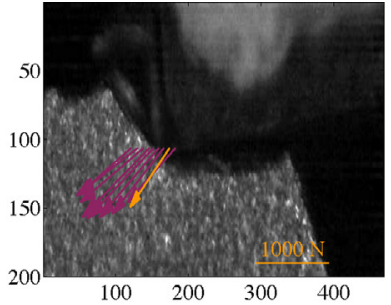

(b) image 10

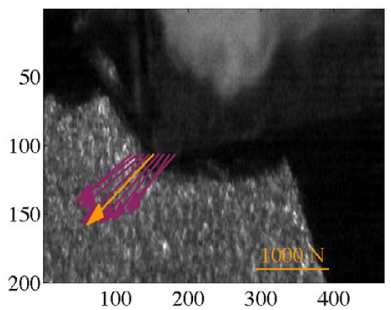

(e) image 13

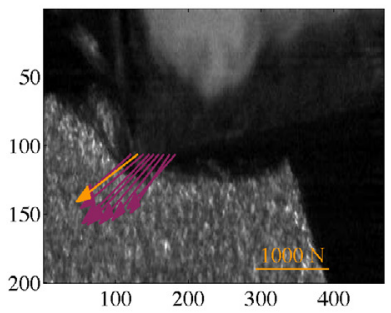

(h) image 16

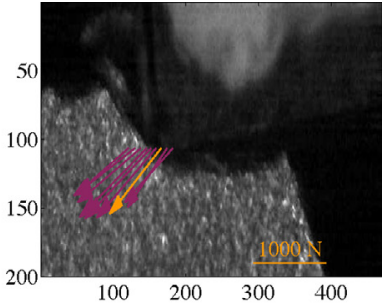

(c) image 11

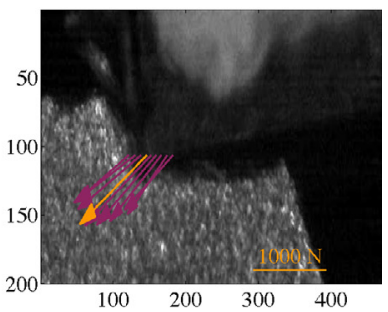

(f) image 14

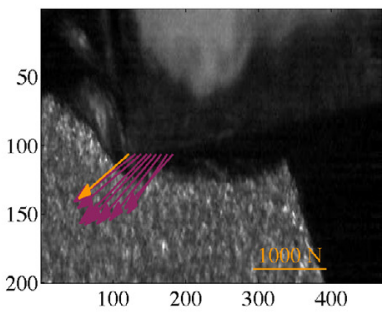

(i) image 17

Fig. 9. Chip segment formation with the current resultant force in orange and the other images forces in purple for (a) image 9 to (i) 17 ; $V_{c}=1 \mathrm{~m} / \mathrm{s}, h=0.10 \mathrm{~mm}$.

and the free surface as seen in image 15 (Fig. 9g). The cutting force then decreases until the segment $n$ starts sliding along the shear plane (see image 16, Fig. 9h). The segment is completely formed and ejected as seen in image 17 (Fig. 9i). The segment formation process is completed. By repeating each steps of the formation, a full chip is formed.

\subsection{Influence of cutting parameters on cutting forces}

Different cutting speed and uncut chip thickness conditions were analyzed thanks to the proposed methodology on films. The obtained mean cutting forces: $F_{C m}$ and $F_{D m}$ and the fluctuation amplitudes: $A_{F_{C}}$ and $A_{F_{D}}$ during segments formation are sum up in Table 1 . The force fluctuation amplitudes increase with the uncut chip thickness whereas the frequency decreases. The cutting speed has only an effect on the segmentation frequency that increases with the speed. The characteristic length of a segment

Table 1

Effect of the cutting speed $V_{c}$ and the uncut chip thickness $h$ on mean cutting forces, fluctuation amplitude due to serrated chip formation and segments formation frequency.

\begin{tabular}{|c|c|c|c|c|c|c|}
\hline \multicolumn{2}{|c|}{ Cutting conditions } & \multicolumn{2}{|c|}{ Mean force } & \multicolumn{2}{|c|}{ Fluctuation } & \multirow{2}{*}{$\begin{array}{l}\text { Frequency } \\
(\mathrm{kHz})\end{array}$} \\
\hline$V_{c}(\mathrm{~m} / \mathrm{s})$ & $h(\mathrm{~mm})$ & $F_{c m}(\mathrm{~N})$ & $F_{D m}(\mathrm{~N})$ & $A_{F_{c}}(\%)$ & $A_{F_{D}}(\%)$ & \\
\hline 1.0 & 0.05 & 533 & 305 & $15 \%$ & $10 \%$ & 9.3 \\
\hline 1.0 & 0.10 & 722 & 409 & $18 \%$ & $22 \%$ & 6.9 \\
\hline 1.0 & 0.15 & 1083 & 682 & $24 \%$ & $18 \%$ & 5.4 \\
\hline 1.5 & 0.10 & 573 & 425 & $18 \%$ & $16 \%$ & 9.7 \\
\hline 2.0 & 0.10 & 830 & 569 & $24 \%$ & $17 \%$ & 13.0 \\
\hline
\end{tabular}

Ls, calculated according to Eq. (16), is not influenced by the cutting speed for $h=0.1 \mathrm{~mm}$, and is $L s=0.15 \mathrm{~mm}$.

$L s(\mathrm{~mm})=\frac{V_{c}(\mathrm{~m} / \mathrm{s})}{f(\mathrm{kHz})}$

\section{Conclusion}

This study has presented a new methodology to measure cutting forces based on the elastic behavior of the machined material. Thanks to two complementary imaging devices (i.e., a double frame imager coupled with laser lighting and a high speed imaging camera with LED lighting), it has been possible to record, at commonly used cutting speed (i.e., between 1 and $2 \mathrm{~m} / \mathrm{s}$ ), multiple pictures of segment formation during hard machining of AISI 52100 steel cut with a c-BN tool. The first device shot two high definition pictures of the cut whereas the second one recorded a full movie of the segment formation but with lower quality.

The relevance of the Flamant-Boussinesq solution as a loading probe in terms of displacement field in the specimen was investigated with an inverse identification procedure to post-process Q4-DIC measurements. The estimated forces by this method are in good agreement (i.e., less than 5\% error) with the mean cutting forces recorded by a piezoelectric sensor. The Flamant-Boussinesq solution was then implemented as displacement basis for an integrated DIC analysis and compared to Q4-DIC measurements. A 4\% relative error on the kinematic field is reached. The mean force estimations of ten trials is close to the mean measured cutting forces but presents higher deviations. The new integrated approach has been validated according to the exposed results. The feasibility of "stress gauging" (Roux et al., 2005) has been shown herein not to 
measure elastic properties but to devise a load cell based on DIC measurements.

The second set-up was utilized to investigate the source of the forces measurement deviations. A whole movie of the cut was processed with the developed method. The cyclic fluctuations of the cutting forces were highlighted and represent about 20\% of the mean measured cutting forces. Furthermore, having both the instantaneous cutting forces and the pictures, it was possible to link them experimentally in order to explain a segment formation. The influence of the cutting speed and the uncut chip thickness on forces fluctuation and segments formations frequency was exposed.

In the near future, the kinematic field and the cutting force measurements will be used for the validation of multiphysical simulations. In addition to thermal field, it can be used for more realistic constitutive equation identification, namely, the cutting test will become the mechanical test itself. Another outlook is to employ the Flamant-Boussinesq solution for surface integrity prediction in the material. Thus, with cutting forces models, the plastically deformed layer induced by the cutting operation could be estimated on a 3D-machined part.

\section{Acknowledgements}

The authors gratefully thank:

- ADEME for the financial support through the WindProcess project;

- NTN-SNR for material supply;

- CEA-Valduc for providing the sCMOS imaging device and laser lighting.

\section{References}

Arriola, I., Whitenton, E., Heigel, J., Arrazola, P., 2011. Relationship between machinability index and in-process parameters during orthogonal cutting of steels. CIRP Ann. Manuf. Technol. 60 (1), 93-96.

Baizeau, T., Campocasso, S., Fromentin, G., Rossi, F., Poulachon, G., 2015. Effect of rake angle on strain field during orthogonal cutting of hardened steel with c-BN tools. Procedia CIRP 31, 166-171.

Besnard, G., Hild, F., Roux, S., 2006. "Finite-element” displacement fields analysis from digital images: application to Portevin-Le Châtelier bands. Exp. Mech. 46 (6), 789-803
Boussinesq, J., 1892. Equilibre d'élasticité - Des perturbations locales que produit au-dessus d'elle une forte charge, répartie uniformément le long d'une droite normale aux deux bords, a la surface supérieure d'une poutre rectangulaire et de longueur indéfinie posée soit sur un sol horizontal, soit sur deux appuis transversaux équidistants de la charge. C. R. Acad. Sci. Paris 114 (1), 1510-1516.

Chou, Y., Song, H., 2003. Thermal modeling for finish hard turning using a new cutting tool. Proc. ASME IMECE 41765, 183-192.

Flamant, A., 1892. Equilibre d'élasticité - Sur la répartition des pressions dans un solide rectangulaire chargé transversalement. C. R. Acad. Sci. Paris 114 (1), 1465-1468.

Grédiac, M., Hild, F., 2012. Full-Field Measurements and Identification in Solid Mechanics. ISTE/Wiley, London, UK.

Hild, F., Roux, S., 2012a. Comparison of local and global approaches to digital image correlation. Exp. Mech. 52 (9), 1503-1519.

Bornert, M., Hild, F., Orteu, J.J., Roux, S., 2012. Digital Image Correlation. John Wiley \& Sons, Inc., pp. 157-190, http://dx.doi.org/10.1002/9781118578469.ch6.

ISO:683-17, 2014. Heat-treated steels, alloy steels and free-cutting steels - Part 17 Ball and Roller Bearing steels, Number 683-17. Afnor.

Mabrouki, T., Rigal, J.F., 2006. A contribution to a qualitative understanding of thermo-mechanical effects during chip formation in hard turning. J. Mater. Process. Technol. 176 (1), 214-221.

Mathieu, F., Leclerc, H., Hild, F., Roux, S., 2015. Estimation of elastoplastic parameters via weighted FEMU and integrated-DIC. Exp. Mech. 55 (1), 105-119.

Movahhedy, M., Altintas, Y., Gadala, M., 2002. Numerical analysis of metal cutting with chamfered and blunt tools. J. Manuf. Sci. Eng. 124 (2), 178-188.

Oxley, P.L.B., 1989. The Mechanics of Machining: an Analytical Approach to Assessing Machinability. E. Horwood, Chichester, England.

Poulachon, G., Moisan, A., 2000. Hard turning: chip formation mechanisms and metallurgical aspects. J. Manuf. Sci. Eng. 122 (3), 406-412.

Pujana, J., Arrazola, P., Villar, J., 2008. In-process high-speed photography applied to orthogonal turning. J. Mater. Process. Technol. 202 (1-3), 475-485.

Ren, H., Altintas, Y., 2000. Mechanics of machining with chamfered tools. J. Manuf. Sci. Eng. 122 (4), 650-659.

Roux, S., Hild, F., 2006. Stress intensity factor measurements from digital image correlation: post-processing and integrated approaches. Int. J. Fract. 140 (1-4), $141-157$.

Roux, S., Hild, F., 2008. Digital image mechanical identification (DIMI). Exp. Mech. 48 (4), 495-508.

Roux, S., Hild, F., Pagano, S., 2005. A stress scale in full-field identification procedures: a diffuse stress gauge. Eur. J. Mech. A: Solids 24 (A), 442-451.

Sutton, M., Orteu, J., Schreier, H., 2009. Image Correlation for Shape, Motion and Deformation Measurements: Basic Concepts, Theory and Applications. Springer, New York, NY, USA.

Umbrello, D., Rotella, G., 2012. Experimental analysis of mechanisms related to white layer formation during hard turning of AISI 52100 bearing steel. Mater. Sci. Technol. 28 (2), 205-212.

Yang, D., Liu, Z., 2015. Surface plastic deformation and surface topography prediction in peripheral milling with variable pitch end mill. Int. J. Mach. Tools Manuf. 91, 43-53. 